Liver, Pancreas and Biliary Tract

\title{
Hepatic encephalopathy in patients with non-cirrhotic portal hypertension: Description, prevalence and risk factors
}

\author{
Valeria Nicoletti ${ }^{a}$, Stefania Gioia ${ }^{a}$, Pierleone Lucatelli $^{b}$, Silvia Nardelli ${ }^{a}$, Chiara Pasquale $^{a}$, \\ Stefano Nogas Sobrinho ${ }^{a}$, Ilaria Pentassuglio ${ }^{a}$, Francesca Greco ${ }^{a}$, Adriano De Santis ${ }^{a}$, \\ Manuela Merli ${ }^{\mathrm{a}}$, Oliviero Riggio ${ }^{\mathrm{a}, *}$ \\ a Department of Clinical Medicine, Center for the Diagnosis and Treatment of Portal Hypertension, “Sapienza" University of Rome, Rome, Italy \\ ${ }^{\mathrm{b}}$ Vascular and Interventional Radiology Unit, Department of Radiological, Oncological and Anatomo-pathological Sciences, "Sapienza" University of Rome, \\ Rome, Italy
}

\section{A R T I C L E I N F O}

\section{Article history}

Received 3 May 2016

Accepted 14 June 2016

Available online 30 June 2016

\section{Keywords:}

Hepatic encephalopathy

Non-cirrhotic portal hypertension

Portal-systemic shunt

\begin{abstract}
A B S T R A C T
Background: Hepatic encephalopathy (HE) is a common complication of cirrhosis but it is less studied in patients with non-cirrhotic portal hypertension (NCPH).

Aims: To describe the prevalence of cognitive impairment (overt and covert HE) in NCPH patients and to identify the risk factors for its development.

Methods: 51 patients with NCPH, 35 with chronic portal vein thrombosis (PVT) and 16 with idiopathic non-cirrhotic portal hypertension (INCPH), were evaluated for the presence of previous or present overt $\mathrm{HE}$ (OHE). The psychometric hepatic encephalopathy score and the SCAN battery were used to detect the presence of covert HE (CHE). 34 compensated cirrhotic patients were used as control. In NCPH patients, abdominal scans were performed to detect the presence of shunts.

Results: None of the patients experienced OHE at evaluation while $5.7 \%$ of PVT and $12.5 \%$ of INCPH patients referred at least one documented episode of previous OHE, similarly to patients with cirrhosis (14.7\%). Even if lower than in patients with cirrhosis (64.7\%), a considerable proportion of patients with chronic PVT $(34.3 \%)$ and INCPH $(25 \%)$ had CHE $(p=0.008)$. The presence of a large portal-systemic shunt was the only factor significantly correlated to cognitive impairment in NCPH patients.

Conclusion: $\mathrm{HE}$ is a tangible complication of NCPH and is mainly related to the presence of portal-systemic shunts.
\end{abstract}

(c) 2016 Editrice Gastroenterologica Italiana S.r.l. Published by Elsevier Ltd. All rights reserved.

\section{Introduction}

Hepatic encephalopathy, at least in the western world, is mainly associated to liver cirrhosis (type C-HE; C from cirrhosis) and is considered a consequence of both the liver damage and the presence of portal-systemic shunts. HE has also been described in animals and in patients with congenital portal-systemic shunts $[1,2]$ (Type B-HE; B from bypass), suggesting that the presence of a large portal-systemic shunt, by reducing the detoxification of gut derived toxins, may induce alterations in the central nervous system even in the absence of significant liver damage. In the western

\footnotetext{
* Corresponding author at: Professor of Gastroenterology, Centro di Riferimento per l'Ipertensione Portale, II Gastroenterologia, Dipartimento di Medicina Clinica, “Sapienza" Università di Roma, Viale dell'Università 37, 00185 Roma, Italy. Tel.: +39 06 49972001; fax: +3906 4453319/49972001.

E-mail address: oliviero.riggio@uniroma1.it (O. Riggio).
}

world, portal-hypertension not due to cirrhosis is an uncommon condition, mainly caused by chronic portal vein thrombosis (PVT). More recently, a disorder characterized by portal hypertension not associated to liver cirrhosis or chronic PVT [3-5] has been better defined $[6,7]$ and named as idiopathic non-cirrhotic portal hypertension (INCPH). Patients with non-cirrhotic portal hypertension (NCPH), as well as cirrhotic patients, may develop collaterals and, in some cases, large spontaneous portal-systemic shunts, which may predispose the patients to HE. In liver cirrhosis the relationship between large portal-systemic shunts and HE is supported by a number of clinical observations such as the high incidence of HE after surgical [8] or radiological [9] porto-caval anastomosis, the relationship between the diameter of the shunt and the severity and recurrence of HE episodes and the amelioration of the neurological symptoms after the reduction of the shunt calibre in patients submitted to transjugular intrahepatic portosystemic shunt (TIPS). Moreover, in cirrhotic patients the presence of large portal-systemic shunts have been related to a severe and persistent 
form of HE, poorly responsive to medical treatment [10], which can be ameliorated by the closure of the shunt $[11,12,13]$.

The aims of the present study were to describe the prevalence of the different grades of cognitive impairment (overt HE and minimal/covert HE) in 51 adult Italian patients with NCPH (35 with chronic PVT and 16 with INCPH) and to identify the risk factors for its development, focusing on the role of large portal-systemic shunts.

\section{Patients and methods}

Among 90 patients affected by NCPH seen and followed up in our Centre for the Study of Portal Hypertension, fifty-one patients available for HE assessment during the study period (from October 2014 to June 2015) were included in the study. Thirty-five were affected by chronic portal vein thrombosis (PVT) and 16 by idiopathic non-cirrhotic portal hypertension (INCPH), according to the diagnostic criteria suggested in the EASL Clinical Practice Guidelines on Vascular diseases of the liver [14].

Portal hypertension was diagnosed on the presence of oesophageal varices or other portal-systemic collaterals and attributed to chronic PVT when imaging techniques (Doppler ultrasound and contrast-enhanced CT-scan) showed the presence of portal cavernoma, defined as the development of a network of tortuous collateral vessels bypassing the obstructive area at hepatic hilum. Neoplastic portal vein obstruction was excluded on the basis of imaging studies and negative tests for serum tumour markers. Cirrhosis was excluded on the absence of causes of chronic liver diseases, normal results of liver function tests and on the absence of advanced fibrosis on liver elastometry. In 8 patients liver cirrhosis and/or obliterative portal venopathy was excluded on the basis of histological features.

In patients with signs of portal hypertension the diagnosis of idiopathic non-cirrhotic portal hypertension (INCPH) was based in all cases on the absence of liver cirrhosis at liver biopsy and on the exclusion of portal and sovrahepatic veins obstruction at Doppler Ultrasound and CT-scan. Other causes of liver disease (chronic viral hepatitis, alcoholic liver disease, non alcoholic steatohepatitis, autoimmune hepatitis, hemochromatosis and Wilson disease) were also excluded by a complete diagnostic clinical and laboratory workout.

Thirty-four cirrhotic patients belonging to the class A of ChildPugh, submitted to the same neuropsychological evaluation, were enrolled as control group. The diagnosis of liver cirrhosis was based on clinical, laboratory and histological features.

Both cirrhotic and NCPH patients were receiving drugs reducing portal hypertension (i.e., $\beta$-blockers) when appropriate (large oesophageal varices); none of the patients was taking statins. No patients were receiving lactulose or rifaximin at the moment of evaluation.

\subsection{HE assessment}

The hepatic encephalopathy assessment included the evaluation of overt HE, previous overt HE and minimal/covert HE.

At enrolment the presence and grade of overt $\mathrm{HE}$, according to West Haven Criteria [15], was evaluated in all patients by a pool of standardized questions as suggested by Amodio et al. [16] and previously described $[17,18]$.

Previous overt HE has been defined as the presence in the patient's history of at least one episode of overt HE (grade II or more), documented in a clinical record of a previous hospitalization.

The presence of minimal/covert HE was assessed by two different batteries of tests [19]: the psychometric hepatic encephalopathy score (PHES) and the Scan battery which explore different domains of cognitive impairment but also have different levels of difficulty: being the scan test more complex than the PHES [20]. PHES includes the digit-symbol-test (DST), the trail-makingtest A (TMT-A) and B (TMT-B), the serial-dotting-test (SDT) and the line-tracing-test (LTT). Each test was scored against age and education-adjusted norms for the Italian population. PHES is the sum of integer scores of each test computed from the adjusted $Z$ values, as follows: score -3 for $Z \leq-3$, score -2 for $-3<Z \leq-2$, score -1 for $-2<Z \leq-1$, score 0 for $-1<Z<1$, score 1 for $Z \geq 1$. A PHES $\leq-4$ was considered abnormal [21]. The Scan battery is a series of 3 computerized tests of increasing complexity, including the simple visual reaction time (SVRT), the choice visual reaction time (CVRT) and the scan test reaction time (STRT) [20]. A total $Z$ score of the whole Scan battery (tZSB) can be calculated and it results from the value of STRT for the patients that are able to perform all the three tests of the battery, from the $Z$ score of CVRT minus 7.5 for those who are unable to perform the sole STRT (the most difficult test of the battery), and from the $Z$ score of the SVRT for the patients who are unable to perform either CVRT and STRT [20]. A total Z SCAN battery $\leq-2$ was considered abnormal.

The tests were administered in a quiet and well-lit room by a hepatologist with expertise on hepatic encephalopathy during a single session of about $45 \mathrm{~min}$; interruption for rest was allowed as soon as fatigue or reduced compliance was suspected. Exclusion criteria were: neuropsychiatric disorders such as dementia (patients with Mini-Mental State Examination <26 were excluded), use of psychotropic drugs, active alcohol intake, language and visual barriers and lack of consent or compliance for the psychometric assessment. Accordingly, 10 patients were excluded from the psychometric evaluation: 2 for language barrier and 8 (all among cirrhotic patients) for active alcohol intake.

The purpose of the study was clearly explained to all the patients before obtaining their written informed consent. The "Sapienza" University of Rome Ethical Committee approved the collection of data of the patients for prognostic studies (Rif.1720/01.10.09).

\subsection{Portal-systemic shunts evaluation}

In 41 of 51 patients with NCPH (28 with chronic PVT and 13 with INCPH), the presence of spontaneous portal-systemic shunts has been evaluated on the basis of contrast-enhanced CT/MRI abdominal scans. The evaluation of $\mathrm{CT} / \mathrm{MRI}$ scans was carried out by a skilled ( 8 year experience) body radiologist ( $\mathrm{PL}$ ), who was blind to the results of the neuropsychiatric assessment. In the presence of shunts, their location and type were evaluated and recorded. Spontaneous portal-systemic shunts have been classified as follows:

- direct splenorenal shunt (Fig. 2, panel A) (tortuous varicosities between the splenic vein and the left renal vein in the absence of varicosities along the gastric wall),

- splenogastrorenal or indirect splenorenal shunt (Fig. 2, panel $\mathrm{B}_{1}-\mathrm{B}_{2}-\mathrm{B}_{3}$ ) (an anastomosis between a short gastric vein, supplying gastric and perigastric varices, and the left renal vein via the left inferior phrenic vein with connection with the splenic vein). In addition to the identification of spontaneous portalsystemic shunts, the presence of iatrogenic shunts (surgical and/or transjugular intrahepatic portosystemic shunt-TIPS) was also evaluated,

- gastrorenal shunt (Fig. 2, panel $\mathrm{C}_{1}-\mathrm{C}_{2}$ ) (tortuous vessels around the stomach that shunts blood towards an enlarged left renal vein without connection with the splenic vein).

\subsection{Statistical analysis}

Data are expressed as mean $\pm S D$, unless specified otherwise. Comparison between 2 groups was performed by chi square test or 


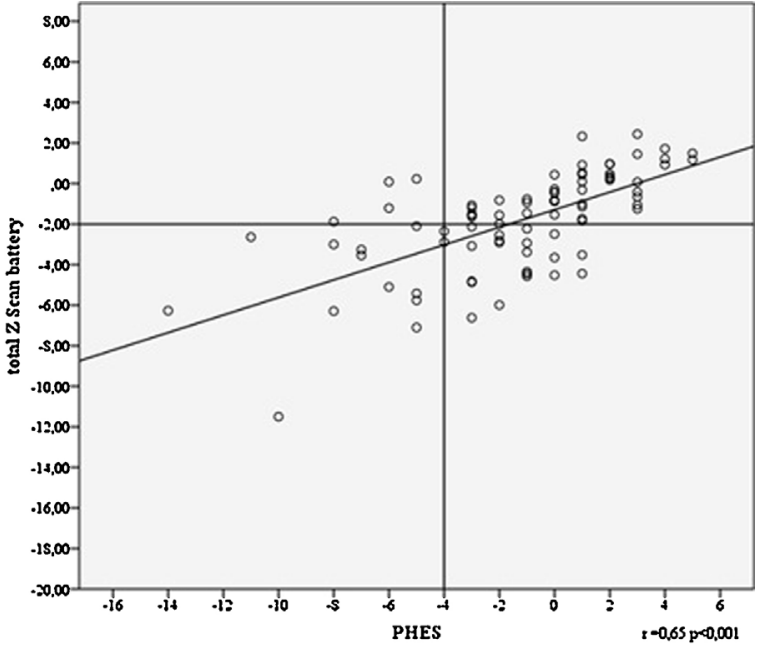

Fig. 1. Correlation between Scan battery and PHES.

unpaired Student $\mathrm{t}$ test, when appropriate. Comparison between 3 groups was performed by analysis of variance (ANOVA) and by Bonferroni multiple comparison post hoc analysis. The relationship between variables was analyzed by Pearson correlations. $p$-Values less than 0.05 were considered significant. The Predictive Analytics Software (PASW) 2009 was used for all computations.

\section{Results}

The demographic, clinical and biochemical features obtained in the patients at entry are summarized in Table 1 . Age was significantly higher in cirrhotic patients than in patients with NCPH $(p<0.001)$. Albumin levels were significantly higher $(p=0.008)$ in chronic PVT group than in the other two, although the mean values were in range of normality in all the three groups.
At the evaluation, signs of portal hypertension (oesophageal varices, variceal bleeding, ascites) were equally present in the three groups ( $p=$ NS). Thirty-eight patients (74.5\%) with NCPH had associated diseases as cause of portal hypertension: prothrombotic disorder (59\%), abdominal surgery/trauma (14\%), immunological disorders (2\%).

The results of the psychometric evaluation are reported in Table 2. Years of education were significantly lower in the cirrhotic group than in the other two groups $(p=0.006)$. Compared to the patients with NCPH, cirrhotic patients had a poorer performance in each test of the PHES and the Scan battery. No difference in psychometric performance was found between the patients with INCPH and chronic PVT.

The total Z-score of the Scan battery (tZSB) was significantly correlated with PHES $(r=0.65 ; p<0.001)$. However, some discordance was observed. In particular, the majority of discrepancy was due to patients with altered Scan and normal PHES although in 3 patients, all in the cirrhotic group, abnormal PHES and normal SCAN was observed. This suggests that not only different levels of difficulty [20] (the scan test is more complex than PHES) but also different domains of cognitive function can be explored by the two group of tests (Fig. 1) [22]. Accordingly, the patients were considered affected by minimal/covert HE when at least one of these two tests was altered.

Table 3 reports the prevalence of minimal/covert HE according to PHES, Scan battery or both as well as the prevalence of previous and overt HE in the patients with cirrhosis, chronic PVT and INCPH. Finally, the prevalence of patients with global cognitive impairment, defined as the number of patients with minimal or overt HE (present or previous) is also reported in Table 3. Cognitive impairment was observed more frequently in cirrhotic patients (67.6\%) then in patients with NCPH, without significant differences between the patients with chronic PVT (37.1\%) and those with INCPH (31.3\%). In all groups the cognitive impairment was mainly represented by subclinical alterations detectable at psychometric testing. In particular none of the enrolled patients showed at the
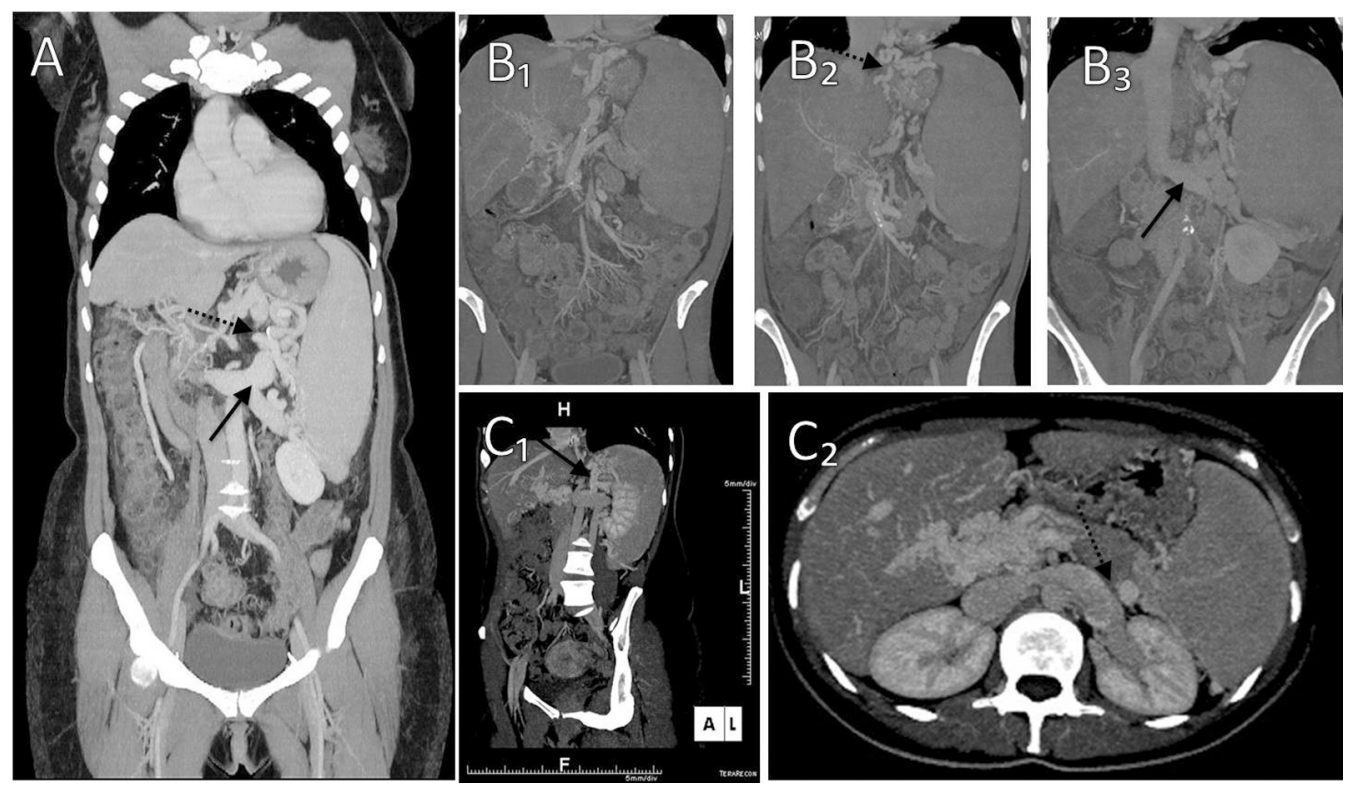

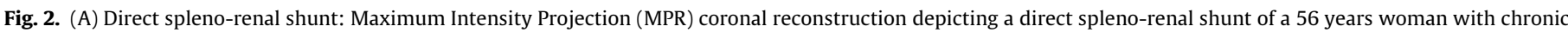

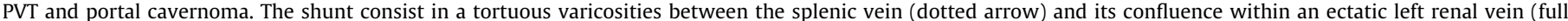

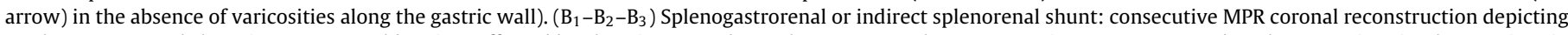

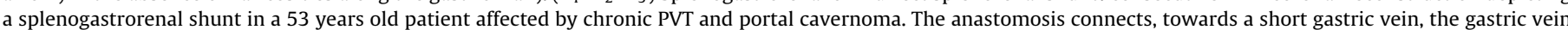

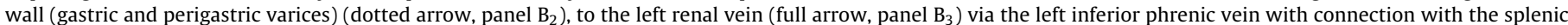

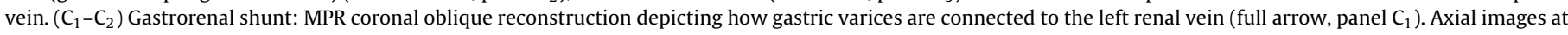
the splenic hilum clearly demonstrate a cleavage plane among the splenic vein (dotted arrow, panel $C_{2}$ ) and the ectatic left renal vein. 
Table 1

Characteristics and clinical features of the patients included in the study.

\begin{tabular}{|c|c|c|c|c|}
\hline & Cirrhosis $(n=34)$ & Chronic PVT $(n=35)$ & $\mathrm{INCPH}(n=16)$ & $p$ \\
\hline Age & $67 \pm 12^{*}$ & $51 \pm 15$ & $53 \pm 9$ & $<0.001$ \\
\hline Sex (male) & $24(70.6 \%)$ & $17(48.6 \%)$ & $10(62.5 \%)$ & NS \\
\hline $\mathrm{HB}(\mathrm{g} / \mathrm{dl})$ & $12 \pm 2^{*}$ & $13 \pm 2$ & $13 \pm 2$ & 0.017 \\
\hline $\operatorname{PLT}\left(10^{3} / \mathrm{mm}^{3}\right)$ & $105 \pm 55$ & $236 \pm 134^{*}$ & $153 \pm 152$ & $<0.001$ \\
\hline Albumin (g/dl) & $3.8 \pm 0.4$ & $4.1 \pm 0.5^{* *}$ & $4.1 \pm 0.4$ & 0.008 \\
\hline Bilirubin (mg/dl) & $1.07 \pm 0.42$ & $1.39 \pm 1.13$ & $1.8 \pm 2.81$ & NS \\
\hline $\mathrm{Na}(\mathrm{mEq} / \mathrm{L})$ & $138 \pm 6^{*}$ & $141 \pm 3$ & $142 \pm 3$ & 0.01 \\
\hline $\mathrm{K}(\mathrm{mEq} / \mathrm{L})$ & $3.95 \pm 0.37$ & $4.38 \pm 1.21$ & $4.56 \pm 0.51$ & NS \\
\hline Creatinine (mg/dl) & $0.9 \pm 0.3$ & $0.8 \pm 0.2$ & $0.9 \pm 0.4$ & NS \\
\hline Variceal bleeding (yes) & $10(29.4 \%)$ & $16(45.7 \%)$ & $6(37.5 \%)$ & NS \\
\hline Esophageal varices: & $18(52.9 \%)$ & $26(74.3 \%)$ & $8(50.0 \%)$ & NS \\
\hline$-F 1$ & $9(26.5 \%)$ & $8(22.9 \%)$ & $4(25.0 \%)$ & \\
\hline$-F 2$ & $5(14.7 \%)$ & $13(37.1 \%)$ & $4(25.0 \%)$ & \\
\hline$-F 3$ & $4(11.7 \%)$ & $5(14.3 \%)$ & $0(0.0 \%)$ & \\
\hline Ascites (yes) & $7(20.6 \%)$ & $3(8.6 \%)$ & $1(6.3 \%)$ & NS \\
\hline Iatrogenic shunt & $6(17.6 \%)$ & $5(14.3 \%)$ & $4(25.0 \%)$ & NS \\
\hline
\end{tabular}

Mean \pm SD; ANOVA and Bonferroni post hoc test

* Significantly different from the other groups.

** Different from cirrhosis.

Table 2

Results of the psychometric evaluation.

\begin{tabular}{|c|c|c|c|c|}
\hline & Cirrhosis $(n=34)$ & Chronic PVT $(n=35)$ & $\mathrm{INCPH}(n=16)$ & $p$ \\
\hline Education (yrs) & $9 \pm 5^{*}$ & $12 \pm 4$ & $13 \pm 5$ & 0.006 \\
\hline MMSE & $28 \pm 1$ & $29 \pm 1$ & $29 \pm 1$ & NS \\
\hline \multicolumn{5}{|l|}{ PHES battery } \\
\hline NCT-A (sec) & $69 \pm 36^{*}$ & $45 \pm 27$ & $36 \pm 13$ & $<0.001$ \\
\hline NCT-B (sec) & $132 \pm 65^{*}$ & $99 \pm 78$ & $84 \pm 47$ & 0.041 \\
\hline $\operatorname{DST}\left(\mathrm{n}^{\circ}\right)$ & $21 \pm 10^{*}$ & $37 \pm 13$ & $38 \pm 11$ & $<0.001$ \\
\hline LTT (sec) & $98 \pm 33^{*}$ & $76 \pm 20$ & $78 \pm 33$ & 0.004 \\
\hline LTT (err) & $59 \pm 71^{*}$ & $23 \pm 19$ & $22 \pm 20$ & 0.004 \\
\hline SDT (sec) & $76 \pm 36^{*}$ & $47 \pm 16$ & $44 \pm 10$ & $<0.001$ \\
\hline PHES & $-3 \pm 4$ & $0 \pm 3$ & $0 \pm 3$ & $<0.001$ \\
\hline \multicolumn{5}{|l|}{ Scan battery } \\
\hline \multicolumn{5}{|l|}{ I subtest } \\
\hline SVRT (sec) & $478 \pm 86^{*}$ & $398 \pm 171$ & $334 \pm 105$ & 0.002 \\
\hline SVRT acc. (\%) & $97 \pm 3$ & $99 \pm 2^{* *}$ & $99 \pm 3$ & 0.044 \\
\hline wSVRT (sec) & $535 \pm 246^{*}$ & $404 \pm 177$ & $340 \pm 116$ & 0.005 \\
\hline wSVRT ( $Z$ score) & $-1.52 \pm 1.53^{*}$ & $-0.43 \pm 1.78$ & $-0.46 \pm 1.44$ & 0.001 \\
\hline \multicolumn{5}{|l|}{ II subtest } \\
\hline CVRT (sec) & $878 \pm 284^{*}$ & $657 \pm 275$ & $566 \pm 201$ & $<0.001$ \\
\hline CVRT acc. (\%) & $98 \pm 4$ & $99 \pm 2$ & $99 \pm 3$ & NS \\
\hline wCVRT (sec) & $897 \pm 289^{*}$ & $663 \pm 274$ & $573 \pm 198$ & $<0.001$ \\
\hline wCVRT ( $Z$ score) & $-2.08 \pm 1.94$ & $-1.24 \pm 1.67$ & $-0.27 \pm 1.71^{* *}$ & 0.007 \\
\hline \multicolumn{5}{|l|}{ III subtest } \\
\hline STRT (sec) & $1921 \pm 301^{*}$ & $1596 \pm 452$ & $1417 \pm 422$ & $<0.001$ \\
\hline STRT acc. (\%) & $70 \pm 26$ & $80 \pm 21$ & $86 \pm 14^{* *}$ & 0.042 \\
\hline wSTRT (sec) & $2524 \pm 775^{*}$ & $1979 \pm 941$ & $1651 \pm 627$ & 0.001 \\
\hline wSTRT ( $Z$ score) & $-2.72 \pm 2.22$ & $-1.59 \pm 2.56$ & $-0.54 \pm 2.10^{* *}$ & 0.008 \\
\hline Total Z Scan battery (tZSB) & $-2.72 \pm 2.22$ & $-1.59 \pm 2.56$ & $-0.54 \pm 2.10^{* *}$ & 0.008 \\
\hline
\end{tabular}

Mean \pm SD; ANOVA and Bonferroni post hoc test.

* Significantly different from the other groups.

** Different from cirrhosis.

Table 3

Prevalence of minimal, previous, overt HE and of global cognitive impairment ${ }^{\mathrm{a}}$ in the patients included in the study.

\begin{tabular}{|c|c|c|c|c|}
\hline & Cirrhosis $(n=34)$ & Chronic PVT $(n=35)$ & $\mathrm{INCPH}(n=16)$ & $p$ \\
\hline Minimal HE (PHES battery) & $11(32.4 \%)$ & $6(17.1 \%)$ & $1(6.3 \%)$ & NS \\
\hline Minimal HE (Scan battery) & $19(55.9 \%)^{*}$ & $11(31.4 \%)$ & $4(25.0 \%)$ & 0.046 \\
\hline Minimal HE (PHES and/or Scan) & $22(64.7 \%)^{*}$ & $12(34.3 \%)$ & $4(25.0 \%)$ & 0.008 \\
\hline Overt HE & $0(0.0 \%)$ & $0(0.0 \%)$ & $0(0.0 \%)$ & NS \\
\hline Previous HE & $5(14.7 \%)$ & $2(5.7 \%)$ & $2(12.5 \%)$ & NS \\
\hline Cognitive impairment & $23(67.6 \%)^{*}$ & $13(37.1 \%)$ & $5(31.3 \%)$ & 0.012 \\
\hline
\end{tabular}

ANOVA and Bonferroni post hoc test.

* Significantly different from the other groups.

a Cognitive Impairment is the number of patients with minimal and/or overt HE (previous or present). 
Table 4

Features of the patients with NCPH with and without cognitive impairment (minimal and/or overt HE).

\begin{tabular}{|c|c|c|c|}
\hline & $\begin{array}{l}\text { Cognitive } \\
\text { impairment } \\
(n=18)\end{array}$ & $\begin{array}{l}\text { No cognitive } \\
\text { impairment } \\
(n=33)\end{array}$ & $p$ \\
\hline INCPH & $5(27.8 \%)$ & $11(33.3 \%)$ & \\
\hline Chronic PVT & $13(72.2 \%)$ & $22(66.7 \%)$ & NS \\
\hline Age & $55 \pm 13$ & $50 \pm 13$ & NS \\
\hline Sex (male) & $9(50 \%)$ & $18(54.5 \%)$ & NS \\
\hline Variceal bleeding & $7(38.9 \%)$ & $15(45.5 \%)$ & NS \\
\hline Ascites & $1(5.6 \%)$ & $3(9.1 \%)$ & NS \\
\hline Oesophageal varices & $12(66.7 \%)$ & $22(66.7 \%)$ & NS \\
\hline Gastric varices & $5(27.8 \%)$ & $9(27.3 \%)$ & NS \\
\hline Portal vein thrombosis & $15(83.3 \%)$ & $24(72.7 \%)$ & NS \\
\hline $\mathrm{HB}(\mathrm{g} / \mathrm{dl})$ & $13 \pm 2$ & $13 \pm 2$ & NS \\
\hline $\operatorname{PLT}\left(10^{3} / \mathrm{mm}^{3}\right)$ & $195 \pm 133$ & $218 \pm 151$ & NS \\
\hline $\mathrm{Na}(\mathrm{mEq} / \mathrm{L})$ & $141 \pm 3$ & $141 \pm 3$ & NS \\
\hline Creatinine (mg/dl) & $0.8 \pm 0.3$ & $0.8 \pm 0.3$ & NS \\
\hline Albumin $(\mathrm{g} / \mathrm{dl})$ & $4.0 \pm 0.4$ & $4.1 \pm 0.5$ & NS \\
\hline Bilirubin (mg/dl) & $1.40 \pm 0.79$ & $1.58 \pm 2.21$ & NS \\
\hline Venous Ammonia $(\mu \mathrm{g} / \mathrm{dl})^{\mathrm{b}}$ & $65 \pm 62$ & $28 \pm 14$ & $<0.01$ \\
\hline Splenomegaly & $13(72 \%)$ & $22(66.6 \%)$ & NS \\
\hline Spontaneous shunts ${ }^{\mathrm{a}}$ & $6(42.9 \%)$ & $4(14.8 \%)$ & 0.047 \\
\hline Splenorenal & $4(28.6 \%)$ & $1(3.7 \%)$ & \\
\hline Gastrorenal & $1(7.1 \%)$ & $3(11.1 \%)$ & \\
\hline Splenogastrorenal & $1(7.1 \%)$ & $0(0 \%)$ & \\
\hline latrogenic shunts ${ }^{\mathrm{a}}$ & $4(28.6 \%)$ & $5(18.5 \%)$ & NS \\
\hline Shunt (spontaneous or iatrogenic) ${ }^{\mathrm{a}}$ & $10(71.4 \%)$ & $9(33.3 \%)$ & 0.020 \\
\hline
\end{tabular}

a CT scan available in 41 out of 51 patients.

b Ammonia was available in 31 out of 51 patients.

evaluation an altered mental status compatible with overt HE but a previous episode of overt HE was documented in an equal number of patients with INCPH (12.5\%), chronic PVT (5.7\%) and cirrhosis (14.7\%). One patient with PVT developed a recurrent type of HE after a TIPS which was refractory to medical therapy and required the reduction of the shunt diameter.

Table 4 reports the characteristics of the patients affected by $\mathrm{NCPH}$, with and without cognitive impairment. The two groups were similar for age, sex, biochemical features and signs of portal hypertension. However, a portal-systemic shunt was more frequently detected in the patients with cognitive impairment than in those without $(71.4 \%$ vs. $33.3 \% ; p=0.02)$. This was due to a higher prevalence of spontaneous portal-systemic shunts ( $43 \%$ vs. $15 \%$, $p=0.047$ ), being the presence of iatrogenic ones similar between the patients with or without cognitive impairment ( $28.6 \%$ vs. $18.5 \%$; $\mathrm{NS}$ ). The direct spleno-renal shunt was the type of spontaneous shunt most frequently detected. Umbilical vein spontaneous shunt was detected in only two patients (one patient with and one without cognitive impairment). Venous ammonia levels were available in 31 out of 51 patients with NCPH and resulted significantly higher in the group of patients with cognitive impairment.

\section{Discussion}

In the present study we analyzed the prevalence and the description of the cognitive impairment (overt and minimal $\mathrm{HE}$ ) detectable in a group of patients with non-cirrhotic portal hypertension which included patients with chronic portal vein thrombosis or with idiopathic non-cirrhotic portal hypertension. A control group of compensated cirrhotic patients (Child-Pugh Class A) was also studied in order to compare the prevalence of cognitive alterations occurring in patients with or without a significant liver damage.

The main results of the study can be summarized as follows: although the prevalence of hepatic encephalopathy (overt or minimal) was significantly lower in the patients with NCPH compared to that observed in the cirrhotic patients ( 32 vs. $67 \% ; p=0.02$ ) a cognitive impairment was detectable in a relevant proportion of patients with non-cirrhotic portal hypertension with no difference between the patients with chronic portal vein thrombosis and INCPH. In patients with NCPH the cognitive impairment was more often subclinical (minimal/covert HE), but about $8 \%$ of the patients experienced at least one episode of overt HE in the past. The main factor associated with cognitive impairment in patients with NCPH was the presence of a large portal-systemic shunt, either spontaneous or iatrogenic, which was detected in $71 \%$ of the patients with alterations in mental state and was likely associated to the increased levels of venous ammonia observed in the group of patients with cognitive impairment. It should be noted that a large shunt was not visible in $30 \%$ of the patients with NCPH and cognitive impairment. As all patients were submitted to extensive examination to exclude neuropsychiatric disorders such as dementia, use of psychotropic drugs and active alcohol intake we are quite sure on the exclusion of obvious cause of cognitive impairment.

Thus, the cognitive impairment of these patients can be considered as an example of type B hepatic encephalopathy, the one occurring in patients without a significant liver dysfunction. We cannot exclude that factors other than large shunt may play a role in determining the cognitive impairment of this kind of patients with well-preserved liver function. However, all the patients are, by definition affected by portal hypertension and probably by some degree of porto-systemic shunting. What we actually need is a method to detect the presence of "functioning shunts" independently on the fact that the shunt is visible at imaging technique.

The characteristic of HE in patients with non-cirrhotic portal hypertension has been described mainly in series of patients collected in the eastern countries and affected by chronic portal vein obstruction. Our results confirm the observation of Sharma et al. $[23,24]$ who detected the presence of subclinical mental state alterations in 35\% of patients with PVT and identified the presence of a large portal-systemic shunt in $67 \%$ of the patients with cognitive impairment. Our study extends these results to the patients with idiopathic non-cirrhotic portal hypertension. This is an infrequent disorder, defined as an intrahepatic portal hypertension in the absence of cirrhosis and extrahepatic portal vein obstruction, which can frequently lead to the formation of large spontaneous shunt [25]. Moreover, in addition to subclinical alterations of the mental status, some patients with $\mathrm{NCPH}$, including those affected by INCPH, can develop signs of overt HE. These clinical manifestations were usually episodic and easily manageable but one of our patients developed recurrent bouts of HE after a TIPS and required the reduction of the shunt calibre to ameliorate [12]. A similar proportion of patients with overt HE has been described in a group of INCPH patients followed up in Spain [26]. Thus, HE occurring in NCPH is characterized by the whole spectrum of manifestations which can be observed in cirrhotic patients. Actually alterations in blood ammonia and cerebral metabolites detectable at magnetic resonance spectroscopy [27], similar to that observable in cirrhotic patients, have been described in patients with portal vein obstruction. The relationship between the presence of a large shunt and the clinical or subclinical manifestations of HE supports the possibility of the closure of the large shunt [11] in patients with recurrent HE refractory to medical treatment.

\section{Conflict of interest}

None declared.

\section{References}

[1] Watanabe A. Portal-systemic encephalopathy in non-cirrhotic patients: classification of clinical types, diagnosis and treatment. Journal of Gastroenterology and Hepatology 2000:15:969-79.

[2] Raskin NH, Price JB, Fishman RA. Portal-systemic encephalopathy due to congenital intrahepatic shunts. New England Journal of Medicine 1964;270:225-9. 
[3] Sarin SK, Sollano JD, Chawla YK, et al. Consensus on extra-hepatic portal vein obstruction. Liver International 2006;26:512-9.

[4] DeLeve LD, Valla DC, Garcia-Tsao G, et al. Vascular disorders of the liver. Hepatology 2009;49:1729-64

[5] Valla DC, Condat B. Portal vein thrombosis in adults: pathophysiology, pathogenesis and management. Journal of Hepatology 2000;32:865-71.

[6] Schouten JN, Garcia-Pagan JC, Valla DC, et al. Idiopathic noncirrhotic portal hypertension. Hepatology 2011;54:1071-81.

[7] Verheij J, Schouten JN, Komuta M, et al. Histological features in western patients with idiopathic non-cirrhotic portal hypertension. Histopathology 2013;62:1083-91.

[8] Huang L, Yu QS, Zhang Q, et al. Transjugular intrahepatic portosystemic shunt versus surgical shunting in the management of portal hypertension. Chinese Medical Journal 2015;128:826-34

[9] Riggio O, Nardelli S, Moscucci F, et al. Hepatic encephalopathy after transjugular intrahepatic portosystemic shunt. Clinics in Liver Disease 2012;16:133-46.

[10] Riggio O, Efrati C, Catalano C, et al. High prevalence of spontaneous portalsystemic shunts in persistent hepatic encephalopathy: a case-control study. Hepatology 2005;42:1158-65.

[11] Laleman W, Simon-Talero M, Maleux G, et al. Embolization of large spontaneous portosystemic shunts for refractory hepatic encephalopathy: a multicenter survey on safety and efficacy. Hepatology 2013;57:2448-57.

[12] Fanelli F, Salvatori FM, Rabuffi P, et al. Management of refractory hepatic encephalopathy after insertion of TIPS: long-term results of shunt reduction with hourglass-shaped balloon-expandable stent-graft. American Journal of Roentgenology 2009;193:1696-702.

[13] An J, Kim KW, Han S, et al. Improvement in survival associated with embolisation of spontaneous portosystemic shunt in patients with recurrent hepatic encephalopathy. Alimentary Pharmacology and Therapeutics 2014;39:1418-26.

[14] EASL Clinical Practice Guidelines: Vascular diseases of the liver. Journal of Hepatology 2015.

[15] Conn HO, Leevy CM, Vlahcevic ZR, et al. Comparison of lactulose and neomycin in the treatment of chronic portal-systemic encephalopathy. A double blind controlled trial. Gastroenterology 1977;72:573-83.
[16] Amodio P, Montagnese S, Gatta A, et al. Characteristics of minimal hepatic encephalopathy. Metabolic Brain Disease 2004;19:253-67.

[17] Ferenci P, Lockwood A, Mullen K, et al. Hepatic encephalopathy-definition, nomenclature, diagnosis, and quantification: final report of the working party at the 11th World Congresses of Gastroenterology, Vienna, 1998. Hepatology 2002;35:716-21.

[18] Cordoba J. New assessment of hepatic encephalopathy. Journal of Hepatology 2011;54:1030-40.

[19] Vilstrup H, Amodio P, Bajaj J, et al. Hepatic encephalopathy in chronic liver disease: 2014 Practice Guideline by the American Association for the Study of Liver Diseases and the European Association for the Study of the Liver. Hepatology 2014;60:715-35.

[20] Montagnese S, Schiff S, Turco M, et al. Simple tools for complex syndromes: a three-level difficulty test for hepatic encephalopathy. Digestive and Liver Disease 2012;44:957-60.

[21] Amodio P, Campagna F, Olianas S, et al. Detection of minimal hepatic encephalopathy: normalization and optimization of the Psychometric Hepatic Encephalopathy Score. A neuropsychological and quantified EEG study. Journal of Hepatology 2008;49:346-53.

[22] Riggio O, Montagnese S, Ridola L, et al. The assessment of covert hepatic encephalopathy: focus on the Scan battery [submitted for publication].

[23] Sharma P, Sharma BC, Puri V, et al. Minimal hepatic encephalopathy in patients with extrahepatic portal vein obstruction. American Journal of Gastroenterology 2008;103:1406-12.

[24] Sharma P, Sharma BC, Puri V, et al. Natural history of minimal hepatic encephalopathy in patients with extrahepatic portal vein obstruction. American Journal of Gastroenterology 2009;104:885-90.

[25] Dhiman RK, Chawla Y, Vasishta RK, et al. Non-cirrhotic portal fibrosis (idiopathic portal hypertension): experience with 151 patients and a review of the literature. Journal of Gastroenterology and Hepatology 2002;17:6-16.

[26] Siramolpiwat S, Seijo S, Miquel R, et al. Idiopathic portal hypertension: natural history and long-term outcome. Hepatology 2014;59:2276-85.

[27] Mínguez B, García-Pagán JC, Bosch J, et al. Noncirrhotic portal vein thrombosis exhibits neuropsychological and MR changes consistent with minimal hepatic encephalopathy. Hepatology 2006;43:707-14. 